05.1

\title{
Закономерности статистического распределения механических свойств волокон полиамида-6
}

\author{
( Ю.М. Бойко ${ }^{1}$, В.А. Марихин ${ }^{1}$, О.А. Москалюк ${ }^{2}$, Л.П. Мясникова ${ }^{1}$, Е.С. Цобкалло ${ }^{2}$ \\ ${ }^{1}$ Физико-технический институт им. А.Ф. Иофффе РАН, Санкт-Петербург, Россия \\ ${ }^{2}$ Санкт-Петербургский государственный университет промышленных технологий и дизайна, Санкт-Петербург, Россия \\ E-mail: yuri.boiko@mail.ioffe.ru
}

Поступило в Редакцию 11 марта 2019 г.

В окончательной редакции 15 апреля 2019 г.

Принято к публикации 16 апреля 2019 г.

\begin{abstract}
Проведен статистический анализ распределения модуля упругости $E$ и удлинения при разрыве $\varepsilon_{b}$ промышленных ориентированных волокон полиамида-6 в рамках моделей Гаусса и Вейбулла. Подтвержден дуализм статистического распределения величин $E$ и $\varepsilon_{b}$ для высокопрочного ориентированного полимера, выявленный ранее для значений прочности $\sigma$ полиамида-6. Он заключается в корректности описания экспериментальных данных при использовании как нормального распределения Гаусса, так и стандартной функции распределения Вейбулла.
\end{abstract}

Ключевые слова: полиамид-6, ориентация, волокна, деформация при разрыве, модуль упругости, статистика.

DOI: 10.21883/PJTF.2019.14.48017.17781

Как известно [1-8], высокопрочные ориентированные полимерные пленки и волокна, а также материалы неорганической природы (стеклянные и кварцевые нити, углеродные волокна и др.) с механической прочностью $\sigma>1 \mathrm{GPa}$ являются хрупкими или квазихрупкими материалами. Поэтому с целью надежного определения статистически выверенного среднего значения их прочности $\sigma_{a v}$ необходимо усреднение результатов гораздо большего числа параллельных испытаний (не менее $50[1,2])$ вместо традиционно используемых трех-пяти. Кроме того, получение большого массива данных является весьма полезным для детализации механизмов разрушения, например, при анализе статистического распределения $\sigma$ сверхпрочных высокомодульных ультраориентированных пленочных мононитей сверхвысокомолекулярного полиэтилена со степенью вытяжки $\lambda=120$, сформованных из гелей $[1,2]$, в рамках модели Вейбулла (Weibull) [3-9]. Нами было впервые показано [10], что данная модель применима для описания не только распределения прочности $\sigma$, но и распределения таких не менее важных механических характеристик, как удлинение при разрыве $\varepsilon_{b}$ (мера пластичности) и модуль упругости $E$ (критерий сохранения формы изделий). Для анализа статистики распределений $\varepsilon_{b}$ и $E$ нами впервые были предложены следующие уравнения:

$$
\begin{aligned}
& \ln \ln \left[1 /\left(1-P_{j}\right)\right]=-m \ln \varepsilon_{0}+m \ln \varepsilon_{b}, \\
& \ln \ln \left[1 /\left(1-P_{j}\right)\right]=-m \ln E_{0}+m \ln E,
\end{aligned}
$$

где $\varepsilon_{0}=\exp (-a / m)$ и $E_{0}=\exp (-a / m)-$ константы, получаемые в результате анализа; $m$ - так называемый модуль Вейбулла; $P_{j}=(j-0.5) / n$, где $j$ и $n-$ порядковый номер образца и общее число измеренных образцов соответственно.
Уравнения (1) и (2) могут быть представлены в упрощенном виде как

$$
y=a+b x,
$$

где $y=\ln \ln \left[1 /\left(1-P_{j}\right)\right], a=-m \ln \varepsilon_{0}$ или $-m \ln E_{0}-$ константы, $b=m, x=\ln \varepsilon_{b}$ или $\ln E$.

Однако справедливость уравнений (1) и (2) требует дальнейшего подтверждения и для других высокопрочных полимерных материалов. Одним из таких материалов, находящим огромное практическое применение, является ориентированный полиамид-6 (ПА-6). Ранее нами [11] впервые был выявлен феномен дуализма статистики распределения прочности ПА-6. Он заключается в возможности описания распределения $\sigma$ как в рамках модели Гаусса, справедливой для физических свойств многочисленных вязкоупругих и пластичных материалов, так и в рамках модели Вейбулла, предложенной для хрупких материалов.

Поскольку статистическое распределение $E$ и $\varepsilon_{b}$ ориентированного ПА-6 не анализировалось на соответствие ни модели Вейбулла, ни модели Гаусса, выяснение возможности проявления подобного дуализма распределения $E$ и $\varepsilon_{b}$ на базе большого числа измерений является актуальной задачей, решению которой и посвящена настоящая работа.

В качестве объектов исследования были выбраны промышленные ориентированные полифиламетные волокна ( 200 моноволокон в пучке) ПА-6 (линейная плотность 213 tex) с прочностью $\sigma_{a v}=0.8 \mathrm{GPa}$, усредненной по результатам 50 измерений. Механические испытания образцов проводились при комнатной температуре на универсальной разрывной машине Инстрон-1122 по методике [11]. Длина образца и скорость растяжения 

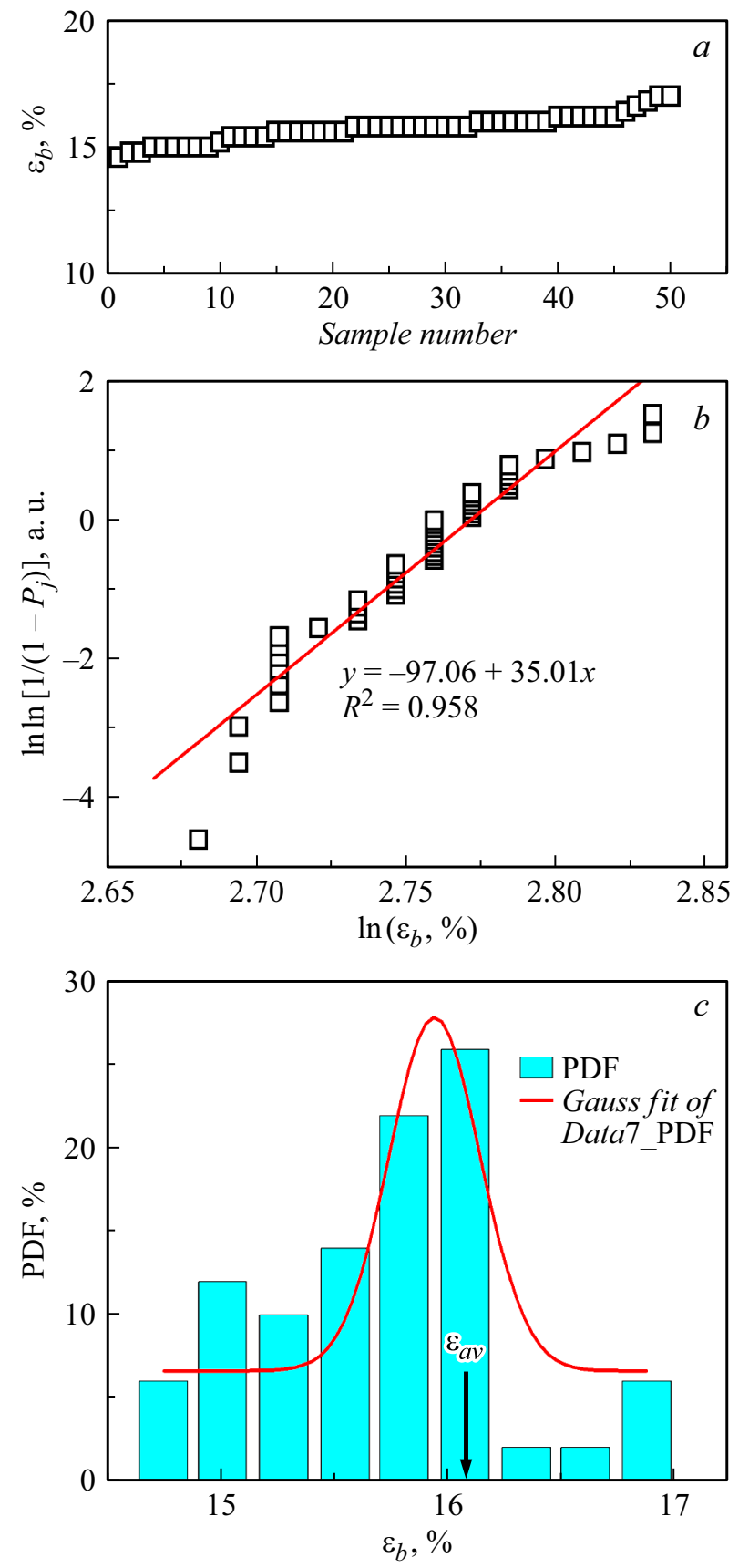

Рис. 1. $a-$ удлинение при разрыве $\varepsilon_{b}$ в порядке возрастания для 50 образцов ориентированных волокон полиамида-6. $b-$ распределение по Вейбуллу удлинения при разрыве $\varepsilon_{b}$ для ориентированных волокон полиамида-6. Прямой линией показан результат анализа методом наименьших квадратов. $c$ - гистограмма распределения (probability density function, $\mathrm{PDF})$ удлинения при разрыве $\varepsilon_{b}$ для ориентированных волокон полиамида-6 и ее аппроксимация функцией Гаусса (сплошная линия).

составляли $500 \mathrm{~mm}$ и $200 \mathrm{~mm} / \mathrm{min}$ соответственно. Исследовалось 50 идентичных образцов.

Проанализируем возможность применения модели Вейбулла для анализа важной механической характери- стики $-\varepsilon_{b}$, отражающей процесс разрушения образца. На рис. 1, $a$ приведены значения удлинения при разрыве $\varepsilon_{b}$ для 50 идентичных образцов полифиламента ПА-6 в порядке их возрастания. Эти данные были проанализированы в рамках стандартной функции распределения Вейбулла в соответствии с уравнениями (1) и (3). Для этих целей они были рассмотрены в координатах $\ln \ln \left[1 /\left(1-P_{j}\right)\right]-\ln \varepsilon_{b}$ (рис. $\left.1, b\right)$ и аппроксимированы линейной зависимостью, проведенной через все экспериментальные точки. Был получен следующий результат: $y=-97.06+35.01 x$ при весьма высоком значении коэффициента детерминации $R^{2}=0.96$, причем рассчитанная по результатам данных рис. $1, b$ величина масштабного фактора $\varepsilon_{0}=16.0 \%$ совпала со средним значением $\varepsilon_{a v}=16.1 \%$, усредненным по результатам 50 параллельных измерений. Следовательно, проведенный анализ статистического распределения $\varepsilon_{b}$ в рамках стандартной функции распределения Вейбулла представляется корректным. Значение $m$ составило 35.01 .

На рис. 1,c приведены результаты анализа данных pис. $1, a$ в рамках модели Гаусса. Видно, что гистограмма функции плотности вероятности в зависимости от $\varepsilon_{b}$, аппроксимированная функцией Гаусса, имеет признаки колоколообразной функции, характерные и для нормального распределения. Несмотря на то что полученная кривая является менее совершенной и более асимметричной, чем аналогичная кривая, полученная для прочности ПА-6 [11], в первом приближении результаты описания $\varepsilon_{b}$ в рамках модели Гаусса также представляются удовлетворительными. Однако результаты этой аппроксимации характеризуются существенно меньшим значением $R^{2}=0.75$ по сравнению с выше проведенной аппроксимацией в рамках модели Вейбулла с $R^{2}=0.96$ для $\varepsilon_{b}$.

На рис. 2, $a$ приведены значения модуля упругости $E$ для 50 идентичных образцов полифиламента ПА-6 в порядке их возрастания. При анализе этих данных в рамках модели Вейбулла на соответствие уравнениям (2) и (3), проведенном на рис. $2, b$, получен следующий результат: $y=-29.55+17.24 x$ при весьма высоком значении $R^{2}=0.94$. Величина масштабного фактора $E_{0}$, рассчитанная по результатам данных рис. $2, b$, составила $5.56 \mathrm{GPa}$, т. е. она практически совпала со средним значением $E_{a v}=5.38 \mathrm{GPa}$, полученным простым усреднением результатов 50 параллельных измерений (разница между $E_{0}$ и $E_{a v}$ составляет всего лишь $3 \%$ ). Следовательно, анализ статистического распределения $E$ с помощью стандартной функции распределения Вейбулла также является корректным. Об этом же свидетельствует совпадение значений $E_{0}$ и $E_{a v}$, а также значений $\varepsilon_{0}$ и $\varepsilon_{a v}$, означающее, что расчетные параметры $E_{0}$ и $\varepsilon_{0}$ имеют физический смысл анализируемых механических характеристик. Значение $m$ составило 17.24.

Проанализируем данные рис. 2, $a$ в рамках модели Гаусса (рис. 2,c). Видно, что гистограмма распределения $E$, аппроксимированная функцией Гаусса, имеет 

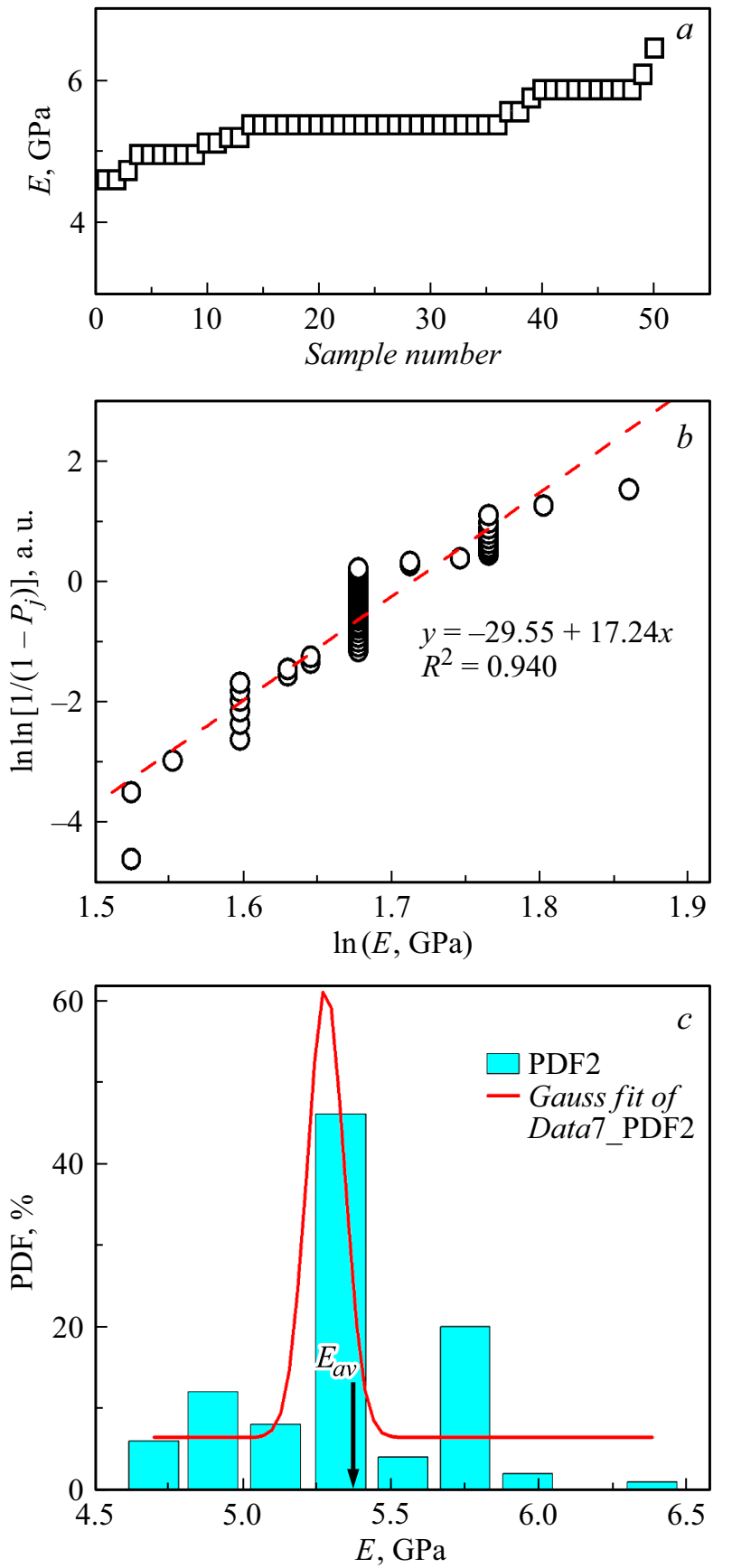

Pис. 2. $a-$ модуль упругости при растяжении $E$ в порядке возрастания для 50 образцов ориентированных волокон полиамида-6. $b-$ распределение по Вейбуллу модуля упругости при растяжении $E$ для ориентированных волокон полиамида-6. Прямой линией показан результат анализа методом наименьших квадратов. $c$ - гистограмма распределения модуля упругости при растяжении $E$ для ориентированных волокон полиамида-6 и ее аппроксимация функцией Гаусса (сплошная линия).

колоколообразную форму. Следовательно, описание распределения $E$ в рамках модели Гаусса можно также считать удовлетворительным, хотя полученная теоретическая кривая характеризуется сушественно меньшей ве- личиной $R^{2}=0.82$ по сравнению с величиной $R^{2}=0.94$, отражающей результаты анализа в рамках модели Вейбулла для $E$.

Однако сопоставление полученных результатов анализа распределений $E$ и $\varepsilon_{b}$ выявляет следующие различия. C одной стороны, описание распределения $E$ в рамках модели Гаусса представляется более корректным, чем описание распределения $\varepsilon_{b}$, так как $R^{2}(E)>R^{2}\left(\varepsilon_{b}\right)$. Кроме того, для $E$ наблюдается резкий максимум для $50 \%$ образцов, совпадающий с $E_{a v}$, тогда как для $\varepsilon_{b}$ кривая распределения является асимметричной с существенным увеличением плотности распределения при $\varepsilon_{b}<\varepsilon_{a v}$. С другой стороны, значение $m=35$, определенное в рамках модели Вейбулла для $\varepsilon_{b}$, является близким к полученному ранее значению $m=43$ для прочности $\sigma[11]$, но оно двукратно превышает значение $m=17$, определенное для $E$. Это означает, что для предельных механических характеристик $\varepsilon_{b}$ и $\sigma$ разброс экспериментальных данных в рамках модели Вейбулла близок и существенно ниже, чем разброс $E$. Иначе говоря, в рамках модели Гаусса вязкоупругие характеристики $(E)$ описываются корректнее, чем прочностные, а в рамках модели Вейбулла, наоборот, описание $\varepsilon_{b}$ и $\sigma$ является более корректным, чем описание $E$. Выявленные различия могут быть обусловлены тем, что из двух исследованных механических характеристик одна $\left(\varepsilon_{b}\right)$ зависит от дефектов структуры, индуцированных деформированием (поэтому она лучше описывается функцией Вейбулла, предложенной для прочностной характеристики), а величина $E$ определяется на начальных стадиях деформирования (доли процента) и в отличие от $\varepsilon_{b}$ слабо зависит от дефектной структуры материала.

Несмотря на то что исследованный ПА-6 является умеренно пластичным материалом $\left(\varepsilon_{b}=16 \%\right)$, его прочностные характеристики удовлетворительно описываются в рамках модели Вейбулла, предложенной для хрупких материалов. Это может быть связано с тем, что процесс разрушения при таких сравнительно невысоких уровнях деформирования контролируется поверхностными дефектами, как и в случае высокопрочных хрупких материалов.

Таким образом, проведенный в настоящей работе статистический анализ распределения модуля упругости $E$ и удлинения при разрыве $\varepsilon_{b}$ для высокопрочных промышленных ориентированных волокон полиамида-6 подтвердил дуализм распределения механических характеристик $\left(E\right.$ и $\left.\varepsilon_{b}\right)$, выявленный ранее для прочности ПА-6 [11]. Он заключается в справедливости для $E$ и $\varepsilon_{b}$ как нормального распределения Гаусса, наиболее часто используемого для анализа погрешности механических характеристик, например, вязкоупругих и пластичных материалов, так и распределения Вейбулла, наиболее часто используемого для анализа механизмов разрушения хрупких материалов. Такое специфическое поведение обусловлено характером деформационного отклика ориентированных полифиламентов ПА-6 на воздействие механического поля, который является промежуточным 
между откликом хрупких и откликом пластичных материалов. Проведенные нами исследования $[1,2,10,11]$ показали, что механические характеристики материала $\left(E, \sigma \quad\right.$ и $\left.\varepsilon_{b}\right)$ могут и должны описываться в разной степени приближения к моделям Гаусса и Вейбулла в зависимости от типа материала (хрупкий, вязкоупругий или пластичный). Оказалось, что для сравнительно жесткого ориентированного ПА-6 более корректными представляются результаты статистической обработки экспериментальных данных в рамках распределения Вейбулла не только для прочности [11], но и для модуля упругости и удлинения при разрыве.

\section{Конфликт интересов}

Авторы заявляют, что у них нет конфликта интересов.

\section{Список литературы}

[1] Бойко Ю.М., Марихин В.А., Мясникова Л.П., Москалюк О.А., Радованова Е.И. // ФТТ. 2016. Т. 58. В. 10. C. 2065-2068. DOI: $10.1134 / \mathrm{S} 1063783416100103$

[2] Boiko Yu.M., Marikhin V.A., Myasnikova L.P., Moskalyuk O.A., Radovanova E.I. // J. Mater. Sci. 2017. V. 52. N 3. P. 1727-1735. DOI: 10.1007/s10853-016-0464-9

[3] Tanaka F., Okabe T., Okuda H., Kinloch I.A., Young R.J. // Composites A. 2014. V. 57. P. 88-94.

DOI: 10.1016/jcompositesa.2013.11.007

[4] Байкова Л.Г., Песина Т.И., Киреенко М.Ф., Тихонова Л.В., Kurkjian C.R. // ЖТФ. 2015. Т. 85. В. 6. C. 83-86. DOI: $10.1134 / \mathrm{S} 1063784215060031$

[5] Wilson D.M. // J. Mater. Sci. 1997. V. 32. N 10. P. 2535-2542. DOI: $10.1023 / \mathrm{A}: 1018538030985$

[6] Sullivan J.D., Lauzon P.H. // J. Mater. Sci. Lett. 1986. V. 5. N 12. P. 1245-1247. DOI: 10.1007/BF01729379

[7] Gurvich M.R., Dibenedetto A.T., Pegoretti A. // J. Mater. Sci. 1997. V. 32. N 14. P. $3711-3716$.

DOI: $10.1023 / \mathrm{A}: 1018603118573$

[8] Barber A.H., Andrews R., Shaudler L.S., Wagner H.D. // Appl. Phys. Lett. 2005. V. 87. N 20. P. 203106.

DOI: $10.1063 / 1.2130713$

[9] Weibull W. // J. Appl. Mech. 1951. V. 18. N 3. P. 293-297.

[10] Boiko Yu.M., Marikhin V.A., Myasnikova L.P., Radovanova E.I. // Colloid Polym. Sci. 2018. V. 296. N 10. P. 1651-1656. DOI: 10.1007/s00396-018-4384-x

[11] Бойко Ю.М., Марихин В.А., Москалюк О.А., Мясникова Л.П., Цобкалло Е.С. // Письма в ЖТФ. 2019. Т. 45. В. 8. C. 37-39. DOI: 10.21883/PJTF.2019.08.47620.17711 